'The kind of doctor I wanted to be.' A qualitative analysis of junior doctors' reasons for choosing to train in psychiatry and in Wales

Alan Slater $^{1 \star}$, Rugi Saeed ${ }^{1}$, Marque Fernando ${ }^{2}$ and Ceri Evans ${ }^{2}$

${ }^{1}$ Cardiff and Vale University Health Board and ${ }^{2}$ Cwm Taf University Health Board

${ }^{*}$ Corresponding author.

doi: 10.1192/bjo.2021.919

Aims. To understand the factors underpinning junior doctors' decision-making processes regarding their choice of psychiatry as a speciality, and why they chose to pursue specialty training in Wales. Background. Over recent years there have been significant challenges in recruiting junior doctors into psychiatry specialty training, both within the UK and in Wales. To counter this a number of measures have been instituted, including advertising campaigns from the Royal College of Psychiatrists ('Choose Psychiatry') and Health Education and Improvement Wales (HEIW) ('Train Work Live'), together with financial incentives. To date there has been no published evaluation of the effectiveness of these measures.

Method. Two focus groups were run (in August 2019 and January 2020) with trainees appointed to new training posts in August 2019. The focus groups featured set questions acting as prompts for discussion. These focused on various factors behind making decisions to train in Psychiatry and choosing to train within HEIW. The focus groups were recorded and transcribed. Following this a thematic analysis was conducted to identify key elements arising from the discussions.

Result. The focus groups were attended by 14 trainees in total (eleven CT1s, four ST4s.) Living in Wales prior to appointment was the most common factor in leading participants to choose to train in Wales, jointly with having a support network (friends or family) in Wales (each present in $57 \%, \mathrm{n}=8$.) Perceptions around a favourable work-life balance were also important $(45 \%, n=5$.) Interactions with staff in an ambassadorial or support role were a significant driver, especially for international medical graduates. Financial incentives and advertising campaigns appeared to have limited influence over participants' decision-making, awareness of these being highest among those already working in psychiatry or in Wales.

Having a foundation year job with a psychiatry placement was a common theme in choosing psychiatry as a specialty $(43 \%, \mathrm{n}=6$.) Work-life balance of the specialty was also important $(21 \%, n=3$.) Again, after these it was hard to identify coherent themes.

Conclusion. We identified three separate groups, namely CT1s, ST4s and international medical graduates, each with distinct themes underlying a range of needs. There was a broad range of factors underlying trainees' decisions which should be reflected when planning future recruitment strategies. It appeared that advertising campaigns and financial incentives were of limited influence.

Melatonin prescribing practices and the provision of sleep hygiene/parent-led sleep behavioural Interventions in S-CAMHS, Aneurin Bevan University Health Board (ABUHB)- Service evaluation as part of quality improvement project

Umer Jalal ${ }^{\star}$, Lokesh Nukalapati, Kathryn Speedy and Megan Davies-Kabir

Aneurin Bevan University Health Board

${ }^{*}$ Corresponding author.

doi: 10.1192/bjo.2021.920
Aims. To identify the number of patients currently on melatonin

To determine the average duration of use of melatonin in patients under the care of S-CAMHS in ABUHB

To investigate whether behaviour interventions were tried and reinforced from time to time

To identify any areas of improvement

Method. Data were collected at St. Cadoc's hospital, in January, 2021. S-CAMHS database was used. Out of total 346 patient currently being managed with pharmacological therapies, 115 (33.2\%) are currently on melatonin. 57/115 were randomly selected as a sample for this this project. Patient notes and EPEX software were also used to collect information regarding the sleep management practices.

Result. During analysis, it was noticed that within the sample, only 46 patients were actively on melatonin. Melatonin is prescribed for sleep related issues in ASD (8/46), ADHD (15/46), ASD and ADHD (10/46), ADHD and mood disorder (0/46), ASD and mood disorder (6/46), ADHD and behaviour difficulties $(2 / 46)$, ASD with behaviour difficulties (1/46), mood disorder (4/46).

$39 / 46$ patients are currently on melatonin for more than a year (85\%). These patients also include 10 patients who have been using melatonin for 5 years or more.

35 patients (76\%) reported improved sleep or some benefit from melatonin.

Evidence for implementation of parent-led sleep behavioural interventions:

Prior to commencing melatonin- Clear evidence available for 35 patients only (76\%). These interventions were however not deemed helpful by most of the service users.

While prescribing melatonin- Clear evidence available for 39 (85\%) patients. Evidence base for melatonin was also discussed during this visit.

During last follow-up visit- Evidence available for 31 patients only (67\%).

Conclusion. Majority of patients under S-CAMHS ABUHB remain on melatonin therapy for longer than one year. Most of these patients have reported benefit from this therapy and preferred to remain on it despite being informed about evidence base for melatonin. Also, there is evidence for implementation of sleep behavioural interventions prior to prescribing melatonin, however their benefit remains unclear.

Recommendations:

The quality of education on sleep hygiene offered should be assessed and improved if needed

Formal group sessions/workshops on sleep hygiene/parent-led sleep behavioural interventions at regular intervals might be useful in reducing the chances of long term polypharmacy or unlicensed drugs

Use of outcome measures such as Child Sleep Habits Questionnaire at intervals can be helpful in identifying any improvement from educational/pharmacological interventions

S-CAMHS database (for patients actively on medications) needs a review and update

\section{Audit of physical health monitoring on admission to Mill Lodge (CAMHS Inpatient Unit, York)}

Andreea Steiu*, Emma Diggins and Nagulan Thevarajan

Mill Lodge, Leeds and York Partnership NHS Foundation Trust ${ }^{\star}$ Corresponding author.

doi: 10.1192/bjo.2021.930

Aims. This audit aimed to evaluate the standard of initial physical health assessment that young people receive on admission to Mill Lodge. 